\title{
Protée
}

\section{L’iconophage mélancolique ou la digestion des images au début du troisième millénaire. Un cas : Timothy McVeigh}

\section{Catherine Mavrikakis}

Volume 29, numéro 3, 2001

Iconoclasmes : langue, arts, médias

URI : https://id.erudit.org/iderudit/030634ar

DOI : https://doi.org/10.7202/030634ar

Aller au sommaire du numéro

Éditeur(s)

Département des arts et lettres - Université du Québec à Chicoutimi

ISSN

0300-3523 (imprimé)

1708-2307 (numérique)

Découvrir la revue

Citer cet article

Mavrikakis, C. (2001). L'iconophage mélancolique ou la digestion des images au début du troisième millénaire. Un cas : Timothy McVeigh. Protée, 29(3), 17-25. https://doi.org/10.7202/030634ar
Résumé de l'article

Cet article analyse le dispositif des images qui présentent l'exécution, par le gouvernement américain, de Timothy McVeigh. Comment met-on en scène la mort d'un condamné ? Quelle est notre relation à la loi et à l'universalité de la justice par le biais des images ? Existet-il une image iconoclaste qui viendrait briser le scénario préfabriqué des représentations d'une exécution annoncée? Quel deuil est-il possible pour les spectateurs mondiaux du scénario de mise à mort d'un assassin ou d'un terroriste? 


\section{L'ICONOPHAGE MÉLANCOLIQUE OU LA DIGESTION DES IMAGES AU DÉBUT DU TROISIÈME MILLÉNAIRE UN CAS: TIMOTHY MCVEIGH \\ CATHERINE MAVRIKAKIS}

À travers le dispositif de mise en images de l'exécution de Timothy McVeigh, quel deuil collectif est-il possible? Les images de l'immeuble éventré d'Oklahoma City ont fait le tour du globe, ont fasciné, ou encore traumatisé le monde entier. La représentation de l'exécution de McVeigh, telle qu'orchestrée par le gouvernement américain, vient-elle consoler les victimes et permettre à tous les spectateurs du monde de faire un deuil quelconque? La mort de McVeigh n'estelle pas représentée à l'avance dans le préfabriqué universel imagier où plus aucune mort ne nous étonne? Quelle image de la justice ou d'une exécution pourrait être iconoclaste? Quelle image pourrait venir briser le convenu des autres, leur construction factice et artificielle? Quelle image pourrait conférer à la mort son caractère non figurable et permettrait en quelque sorte de commencer un deuil réel? Quelle image nouvelle pourrait bien venir se glisser dans le film de l'exécution et dans son "making of»? Quelle image permettrait aux iconophages que nous sommes de sortir réellement de leur mélancolie et de pouvoir faire notre deuil? Devons-nous avec Freud nous demander si une image est réellement dotée d'un tel pouvoir?

L'exemple de McVeigh et de l'interdiction de la représentation de sa mort devrait nous convaincre que certaines images sont construites comme potentiellement iconoclastes, comme potentiellement perturbatrices du système légal étatique. Mais de telles images n'existent peut-être pas. Il faut plutôt voir que le fonctionnement de notre système d'images se fonde sur la construction fictive d'une iconoclastie par l'image elle-même. Des images mythiques iconoclastes, voilà peut-être ce qui vient fonder notre rapport à l'image, sans qu'ait jamais été faite la preuve qu'un mot ne vaut pas mille images, que le mot n'est pas, en dernière instance, ce qui reste iconoclaste. Nous croyons que l'analyse du cas McVeigh permet de comprendre l'enjeu de la fiction de tout iconoclasme visuel à l'ère des images.

\section{LA CONSTRUCTION D'UNE IMAGE PRIVÉE}

Le 19 avril 1995, à Oklahoma City, on s'en souvient peut-être, un édifice fédéral américain était la cible d'un attentat terroriste. Cent soixante-huit personnes perdirent la vie dans l'explosion de l'immeuble. Le responsable de ce 
massacre, Timothy McVeigh, devait après jugement être exécuté le 16 mai 2001, à 7 heures du matin, par injection, mais comme un document de 3100 pages n'avait pas été remis aux avocats de la défense par le FBI, l'exécution, au moment où j'écris ces lignes, a été reportée au 11 juin 2001. Ce qui m'intéresse ici, ce n'est pas de savoir si McVeigh a été exécuté ou non. La conclusion à cette histoire a peu d'importance pour mon propos. La mise en scène et en images de cette exécution orchestrée par le gouvernement fédéral américain me semble devoir davantage retenir l'attention, parce qu'elle permet de comprendre, d'une part, le rôle politique actuel de la production d'images de la justice et, d'autre part, de penser, dans cet espace de l'image contrôlé par les instances fédérales des États-Unis, une ou des images qui ne seraient pas des images officielles, des images préfabriquées d'une exécution toujours annoncée. La question est: comment est-il possible de faire voler en éclats ou de miner l'institution de l'image de la justice qui est en train de se refonder sous nos yeux?

Le dispositif des images est ici très complexe. En effet, un grand nombre de survivants à l'attentat et des membres des familles des victimes ont demandé à assister à l'exécution, de sorte qu'il a fallu procéder à un tirage au sort afin de déterminer quels seraient les témoins de l'exécution. Sept survivants ont été choisis de cette manière, ainsi que trois parents ou proches des victimes. Ceux qui n'ont pas eu le privilège de pouvoir assister à l'exécution ont dû se contenter d'en être les spectateurs à distance, à défaut d'être les témoins oculaires. Car on projette de filmer et de diffuser en circuit fermé la mise à mort du condamné pour le bénéfice de 2300 personnes environ, survivants ou proches des victimes, pourvu qu'ils aient réservé un siège avant le $1^{\mathrm{er}}$ mai.

Néanmoins, le spectacle s'arrêtera là. Aucune caméra, aucune enregistreuse, aucun téléphone cellulaire ne seront permis sur les lieux de l'exécution et sur les lieux de sa diffusion. Nul autre, dans le monde entier, ne pourra observer l'exécution du coupable. Un juge fédéral en a décidé ainsi et a refusé de laisser projeter sur Internet les images en direct de la mort de McVeigh. De toute façon, la loi américaine interdit de reproduire (audio-)visuellement toute exécution fédérale. Si l'on permet à certains de pouvoir voir la retransmission de l'exécution, il s'agit là d'un précédent: c'est que trop de personnes étaient impliquées pour pouvoir assister toutes à l'exécution sur place. C'est sur cette permission gouvernementale de voir les images de la mort de façon sélective, non universelle, que je voudrais m'attarder.

Car cette diffusion en circuit fermé d'une exécution par l'État soulève de nombreux problèmes. Depuis 1963, il n'y a eu aux États-Unis aucune exécution commandée par l'État fédéral.

La façon dont le gouvernement américain a traité la mise en scène et en images de cette condamnation à mort revêt ici une importance toute particulière. En ce qui concerne une réflexion sur les images modernes, l'exemple de la diffusion de la mort de McVeigh est tout à fait surprenant. Pour le dire rapidement, disons que dans un monde globalisant où les images sont en surabondance, où il est difficile de faire la distinction entre les images d'une guerre réelle et des images de films, dans un monde où une nouvelle ne suscite qu'un sentiment de déjà-vu, de répétition, où le sujet ne peut être que blasé, comme l'explique Virno dans Le Souvenir du présent. Essai sur le temps historique, l'idée que des images circuleraient uniquement de façon à n'être présentées qu'à ceux qui sont concernés mérite d'être analysée.

Les images de l'exécution de McVeigh n'ont pas été présentées dans le but d'informer le monde que justice a été rendue, puisque cela aura été fait dans le discours de l'État. Ce dont il s'agit, c'est de faire des spectateurs de l'exécution des témoins et de mettre tous ceux qui verront la scène à la place de ceux qui y seront. Ouvrir la diffusion de la mise à mort à un large public sur Internet permettrait à des "voyeurs", des «curieux", des gens «non concernés» d'assister à un spectacle. Ici, pour le juge, il y a une nécessité éthique de donner à voir des images uniquement à ceux que ces images concernent... McVeigh pourra donc demander lui-même à six proches d'assister à son exécution, puisque, en quelque sorte, le spectacle 
de mise à mort ne peut être vu que par des familiers. Gore Vidal, correspondant de McVeigh depuis l'attentat, assistera donc à l'exécution. Dans le cas qui nous intéresse, la justice ne fonctionne pas comme un dispositif d'images justes ou édifiantes qui circuleraient pour tous. Je veux dire que l'idée générale de la loi et de son exécution pourrait être accessible à tous et à toutes, comme principe de la loi universelle. En effet, on pourrait imaginer que dans notre monde moderne, l'image pourrait venir montrer l'universel de la loi, en tenir lieu et devrait rendre à l'exécution publique son caractère exemplaire, collectif et fondateur. On serait en droit de croire que la mise à mort devant Internet aurait permis à tous et à chacun, à travers le monde, de se rappeler que nul n'est censé ignorer la loi et que le crime peut et doit être puni, que l'État est là pour cela. C'est le scénario le plus plausible, celui que l'on était déjà en train de mettre en place dans nos imaginaires dans une époque d'accélération de la surprésence des images, dans une ère où l'idée de punir les auteurs de crime contre l'humanité grâce à un tribunal international existe et est médiatisée. C'est le scénario auquel on pouvait s'attendre, surtout, lorsque le Président des États-Unis a parlé de McVeigh comme de l'homme qui justifie le plus au monde la peine de mort. Il est le criminel exemplaire, le méchant universel, celui qui serait le plus en droit de mourir devant le monde entier. Si McVeigh est l'exemple du condamné à mort, s'il légitime la loi, pourquoi, logiquement, n'a-t-il pas droit à ce que les images de sa mort s'inscrivent comme la représentation de la justice universelle américaine?

Mais la scène à laquelle nous nous attendions n'aura pas lieu. L'exécution de Timothy McVeigh ne peut servir d'exemple national, juridique ou encore universel. Les images de l'exécution d'un terroriste n'appartiennent qu'à ceux que celui-ci a blessés, qu'aux personnes à qui il a fait subir un tort (parents ou proches des victimes, survivants de l'attentat). Sa mort n'est donc pas ce qui permettrait au particulier de l'affaire de se hisser au niveau du général. Le film de l'exécution de McVeigh est, en quelque sorte, propriété privée, tout comme l'acte du terroriste que l'on vide ici de tout contenu idéologique, de toute valeur politique. Il est à noter que Timothy $\mathrm{McVeigh}$, ancien soldat de la Guerre du Golfe, a lui-même demandé à ce que sa mort soit diffusée à la télévision. Lui-même voulait faire de sa mort un symbole universel de l'injustice de la justice. McVeigh a compris l'exemplarité de sa mort et a désiré, par la diffusion de son exécution, montrer la folie de la justice du gouvernement américain, à laquelle il s'est attaqué. Je ne cherche pas ici à légitimer la cause d'extrême-droite de McVeigh, ni à penser son attentat. Ce que je tente de montrer, c'est l'exclusion du dispositif spectaculaire de certaines images de la mort, celles de l'accusé lui-même. Cette exclusion vient briser une logique de mise en scène de justice universelle ou de représentation organisée par le gouvernement américain. McVeigh, en voulant que sa mort soit diffusée, désirait que son acte ne soit pas vidé de son sens, que sa punition soit exemplaire et témoigne, pour lui, devant tous, de l'injustice de l'État. Or l'État doit se défendre contre cette exemplarité que McVeigh veut mettre en scène. L'État lui refuse le droit à se donner comme héros ou victime de la loi fédérale, ou de la loi tout court. McVeigh a tué et blessé des humains et, si justice il y a, elle doit se soumettre à la loi du talion: «œil pour œil, dent pour dent». Les témoins peuvent alors devenir des acteurs de la scène de la mise à mort-revanche: si McVeigh a tué mon enfant, je peux me faire croire que je tue cet homme, par l'intermédiaire du dispositif judiciaire légal. De par sa décision - qui exclut le reste du globe de la vision de l'exécution par l'État d'un terroriste -, le juge fédéral qui a statué sur le cas permet la mise en place d'une vengeance des personnes concernées par l'attentat. Il ne s'agit donc pas de l'accomplissement de la justice - ce que, malgré tout et assez terriblement, l'exécution mise en images aurait pu peut-être imaginairement permettre -, mais plutôt d'une politique visant à rendre à chacun la monnaie de sa pièce. Le monde entier exclu des images de l'exécution devient le spectateur d'une nouvelle scène, une scène au second degré, la scène où McVeigh est exécuté devant «ses» 
victimes et ses proches, eux aussi personnes privées. Or, l'attentat commis par McVeigh ne visait personne en particulier, il s'attaquait à une instance générale, à une institution américaine: le gouvernement fédéral. Ce dont il est question ici, c'est de réduire tous les actes posés par McVeigh et par l'État sur sa personne à des actes singuliers ne relevant d'aucune visée transcendante. Les images de sa mort restent privées et font de son acte une attaque contre quelques personnes qui le punissent par le biais de l'image.

Il semble que l'État américain n'est pas certain du sens qui serait donné aux images diffusées mondialement de l'exécution de McVeigh, même s'il contrôlait totalement la présentation de ces images.

Tout se passe comme si les images de la mort du criminel exemplaire pouvaient donner la sensation au monde entier d'avoir devant les yeux la victime exemplaire et l'injustice exemplaire. Car que verrionsnous si McVeigh était tué devant nos caméras mondiales? On verrait l'État qui inflige la mort. On verrait la mort donnée à quelqu'un qui ne peut se défendre. À moins que dans un coin de l'image, on ne fasse sans cesse des gros plans sur le visage des victimes de McVeigh, qui viennent en quelque sorte permettre l'exécution, la légitimer. À moins que dans un coin de l'image, on ne nous montre sans cesse le bâtiment en flammes, avec les agonisants qui crient. Et même... Comme le dit Hegel, dans son Introduction à l'esthétique sur la représentation en art:

Qu'une bonne morale puisse être déduite d'une représentation concrète, de la représentation d'un événement, c'est ce qu'on peut admettre de façon générale. Tout dépend de l'interprétation, car en ce qui concerne la morale il y a peu de choses ou de faits dont on ne puisse tirer une morale. On a défendu et excusé les représentations artistiques et les ouvres littéraires les plus immorales, sous le prétexte que, pour pouvoir reconnaître le bien, on doit savoir ce qui est le contraire du bien, et c'est ainsi qu'on a cru pouvoir justifier l'immoralité dans l'art. Ce qui n'a pas empêché de dire que les représentations de MarieMadeleine, la belle pécheresse, ont induit en péché plus d'hommes qu'elles n'ont suscité de repentirs; mais ne faut-il pas avoir péché pour pouvoir se repentir? L'exigence morale a ici un caractère trop général, trop vague. (Hegel, 1979: 49-50)
La morale ici, tout comme la justice, ne pourrait se déduire d'elle-même à partir des images de la mort de $\mathrm{McVeigh}$. On ne sait jamais où les représentations de la mort de McVeigh pourraient conduire en ce qui concerne une interprétation morale ou une position de la justice. On ne sait pas si ces images ne pourraient pas être vues comme le contraire de ce qu'elles prétendent montrer. En effet, beaucoup de gens qui militent contre la peine de mort désirent que ces images soient diffusées mondialement, afin de montrer la violence de l'État sur les corps. De même, de nombreuses associations d'extrême-droite catholique souhaitent la diffusion de l'exécution afin de dissuader tout éventuel criminel. Il n'est pas possible de prévoir le sens donné aux images de la mort en direct de McVeigh. Néanmoins, on peut se demander ce que nous verrions lors de cette exécution que nous n'avons pas vu mille fois? N'avons-nous pas vu mille morts à la télévision, mille exécutions par l’État? Mille vidéoclips où les morts réelles et les morts fictives se côtoient, sans que nous sachions ni même voulions distinguer le vrai du faux? Est-ce que l'exécution de McVeigh viendrait briser le contrôle étatique ou médiatique des images qui de toute façon n'étonnent plus personne? Il est impossible de croire que la mort en direct de McVeigh sur un réseau mondial viendrait donner du souffle à de nouvelles révolutions... Aucune image de nos jours ne nous choque. Et le petit Palestinien traqué dans un coin d'une rue et qui se fait tuer en direct sous les yeux de son père, alors que les caméras tournent, n'a occupé notre attention qu'un jour ou deux. Dans le déluge des images préfabriquées, nous avons déjà tout vu, même si nous ne voyons rien car rien ne nous surprend. Et c'est précisément cela le problème: le gouvernement américain, tout comme le dit Hegel, sait qu'il n'y aurait aucune morale universelle qui émanerait de l'image de l'exécution diffusée mondialement. Le prêt-à-porter des images de la mort de McVeigh ne conférerait aucune morale à la scène. Les images ne nous atteindraient pas, de toute façon. Aucune nécessité de prise de position claire n'en sortirait, aucun engagement pour la justice, quelle que 
soit notre conception de celle-ci, n'aurait vraiment lieu. Les images, nous les connaissons toutes à l'avance et elles ne nous mobilisent absolument plus. Mais, dans la diffusion universelle de la mort de McVeigh, rien de la justice ne pourrait se déduire en soi. Même si les images de la mort de McVeigh risquaient très certainement de nous laisser indifférents, leur interprétation quant à la morale et à la justice ne serait pas contrôlable, ne serait pas contenue en elles. Ce que l'État américain peut faire, c'est simplement diriger nos regards, fabriquer une scène pour que nous voyions une simili-justice, celle de la loi du talion. Ce que le public mondial doit voir, ce sont les victimes regardant l'exécution de McVeigh. Ce sont donc ici les victimes qui tuent, qui demandent la mort. Le gouvernement veut mettre en place un dispositif d'images où est vue simplement la revanche des victimes. Il ne veut en aucun cas que McVeigh, par les images, puisse devenir la victime du gouvernement. Il doit rester celle de ses victimes vengeresses, c'est-à-dire des personnes moralement habilitées à le tuer ou à le faire tuer.

Le fait que le gouvernement ne veuille pas diffuser universellement cette mort donne raison à la folie paranoïaque de McVeigh qui pense qu'il y a un complot du gouvernement américain contre ses citoyens. En termes plus normaux, ou encore plutôt névrosés, cette mise en images de la mise à mort montre le contrôle exercé par l'État américain sur ce type d'images, ce qui ne nous étonne même plus. Il n'y a qu'un paranoïaque, comme McVeigh, pour s'en étonner encore. Si McVeigh est dans le discours du Président le criminel universel, le condamné à mort exemplaire, les images pourraient tourner, malgré un dispositif très contrôlé, en la défaveur du gouvernement américain, car, dans les images, $\mathrm{McVeigh}$, au moment de sa mort, pourrait être une victime aux yeux de la planète. Il deviendrait un homme que l'État tue et les spectateurs risqueraient de ne pas s'identifier aux victimes blessées par l'attentat d'Oklahoma City, mais plutôt de ne voir que l'horreur de la mort. Il y a donc un hiatus, une différence entre la représentation des images de l'exécution et le discours sur celle-ci. Il faut, dans cette mise en scène de l'exécution, tourner tous les regards vers le regard des victimes qui, elles, auraient, comme nous l'avons dit, le droit de demander la mort de McVeigh et d'y assister.

On peut penser à Adorno qui dans Minima

Moralia écrivait:

Au cinéma, les actualités: prise de l'archipel des Mariannes et notamment de lîle de Guam. L'impression qui s'en dégage n'est pas qu'on livre des combats mais qu'on procède à des travaux mécanisés de dynamitage d'infrastructures routières à grande échelle et avec une énergie incroyable ou encore qu'il s'agit d'«enfumer» et d'exterminer les insectes à l'échelle planétaire. On mène les opérations jusqu'au point où il ne reste plus ancune végétation. L'ennemi est dans le rôle d'un patient et d'un cadaure. Comme le juif sous le nazisme, il ne fait plus l'objet que de mesures administratives et techniques: et quand il se défend, ses actions défensives ont d'emblée le même caractère. (Adorno, 1983: 54)

Ce qu'Adorno révèle c'est que les images de la guerre et de la mort ont été vidées, dans la présentation qu'on en a faite, de leur contenu violent, pour devenir des images qui reflètent une efficacité administrative et technique. Cette efficacité qu'Adorno dénonce, on la retrouve dans le discours et dans un texte de 56 pages, un protocole fédéral d'exécution qui prévoit les scènes de la mort de McVeigh jusque dans les moindres détails, jusqu'au dernier repas du condamné et jusqu'à la manière de conserver le poison qu'on lui injectera. Dans le discours, dans l'écrit, il est donc possible de préserver le côté administratif de l'affaire, comme c'était le cas des actualités cinématographiques dont parle Adorno. Il semble que l'image, elle, ne permettrait pas de mettre en scène la seule machine administrative. Sur la pellicule de la mort, il pourrait y avoir le visage de McVeigh mourant. Qui sait comment on pourrait interpréter cela? Comment interpréter un visage? En ce sens, le visage de la mort que prendra McVeigh pourrait être iconoclaste. C'est une possibilité. Ce n'est pas qu'il viendrait assurément briser toutes nos conceptions, préjugés ou convictions sur la peine de 
mort, mais il viendrait montrer le particulier de la loi américaine. Il pourrait très littéralement incarner la justice, mais aussi son horreur. Le visage de McVeigh devrait à lui seul assumer toute l'exécution de la justice universelle. Son visage pourrait devenir le sceau de la loi et dire: "voici la loi, celle de la mort». Or, aucun visage au monde ne peut prendre sur lui, dans sa particularité, la totalité de la loi. La justice doit être aveugle et ne peut avoir les yeux de McVeigh mourant. Quelque chose de la loi et du visage excèdent la représentation Or, McVeigh prétend que son visage mourant serait celui même de l'injustice incarnée. De l'image de son visage, on pourrait donc déduire aussi la violence terrorisante de l'État.

Du visage de McVeigh, on pourrait conclure beaucoup de choses imprévisibles, incontrôlables et même opposées à celles dont on désirait convaincre. Il n'y a pas de garantie sur ce que l'image pourrait dévoiler. En ce sens, l'interprétation de ce visage est par nature arbitraire et ne se déduit absolument pas de l'image elle-même.

\section{TÉMOINS, SPECTATEURS ET MANGEURS D'IMAGES}

Dans ce contexte - le contexte du témoin oculaire de l'image -, il faut se demander pourquoi toutes les victimes ne sont pas condamnées à voir l'exécution de leur coupable par le biais d'images vidéo. Pourquoi certains peuvent et doivent être présents, en chair et en os sur les lieux du "crime»? On trouverait éventuellement un début de réponse à cette question dans la perte de confiance que nous avons dans les images, dans leur authenticité ou leur importance. En Colombie, on a proposé de présenter le journal télévisé en noir et blanc, afin que les téléspectateurs puissent faire la différence entre des images de fiction - qui sont en couleurs - et les images d'informations sur la réalité. On tentait ainsi de permettre aux spectateurs de retrouver, face aux images, un lieu d'authenticité. Grâce au noir et blanc, grâce à la forme adoptée, on crée un effet de réel.

Des victimes doivent donc assister au spectacle de l'exécution du coupable afin de permettre aux images auxquelles les autres victimes auront accès d'être authentifiées. Il s'agit de ce que j'appellerais le syndrome "Capricorn One»: dans un film portant ce titre, des astronautes américains s'aperçoivent que la prétendue conquête spatiale est une supercherie. Personne ne serait jamais allé sur la lune et la NASA ment au peuple américain comme aux astronautes en créant des images fictives de l'espace. Dans le cas McVeigh, les témoins choisis au hasard permettront de donner une vérité aux images de l'exécution, dans un monde où de telles images sont constamment fabriquées et mises en scène. Mais il y a plus à penser dans cette présence des victimes lors de cette mise à mort.

Dans Le Différend, Lyotard cite le révisionniste Faurisson sur la question des chambres à gaz.

J'ai analysé des milliers de documents. J'ai inlassablement poursuivi de mes questions spécialistes et historiens. J'ai cherché, mais en vain, un seul ancien déporté capable de me prouver qu'il avait réellement vu de ses propres yeux, une chambre à gaz. Et Lyotard de poursuivre:

Avoir «réellement vu de ses propres yeux» une chambre à gaz serait la condition qui donne l'autorité de dire qu'elle existe et de persuader l'incrédule. Encore faut-il prouver qu'elle tuait au moment où on l'a vue. La seule preuve recevable qu'elle tuait est qu'on en est mort. Mais, si l'on est mort, on ne peut témoigner que c'est du fait de la chambre à gaz. Le plaignant se plaint qu'on l'a trompé sur la situation dite solution finale. Son argument est: pour identifier qu'un local est une chambre à gaz, je n'accepte comme témoin qu'une victime de cette chambre à gaz; or il ne doit y avoir, selon mon adversaire, de victime que morte, sinon cette chambre à gaz ne serait pas ce qu'elle prétend; il n'y a donc pas de chambre à gaz. (Lyotard, 1983: 6-17)

Les victimes de McVeigh et les quelques proches de celui-ci qui assisteront sur place à sa mort seront en quelque sorte ceux qui sont allés à la chambre de mort avec lui et qui sont revenus pour témoigner. La logique du gouvernement américain est ici très proche de celle d'un Faurisson, c'est-à-dire que ceux qui pourront dire que $\mathrm{McVeigh}$ est mort réellement seront seulement ceux qui sont victimes comme McVeigh. Tout se passe comme si on allait faire des "condamnés à mort» - que sont les victimes de McVeigh et les proches de celui-ci 
(qui sont vues comme victimes de cette histoire ${ }^{1}$ ) - des individus présents dans la chambre de mort. Ceux-ci reviendront du sein des morts ou de la mort pour témoigner et dire que McVeigh est réellement mort, que la machine de la justice américaine est authentique et a bien fonctionné. La présence des victimes sur le lieu de l'exécution vient authentifier celle-ci, mais révèle une troublante évidence de la logique sousjacente, à savoir que seules les victimes, celles qui auront été au plus proche de la mort, dans l'attentat et dans l'exécution, peuvent témoigner. Ce qui disparaît dans l'opération, c'est le témoin universel, celui qui peut témoigner en toute bonne foi et en vérité, non pas parce qu'il est victime, mais parce qu'il est humain et que rien d'humain ne lui est étranger.

Dans L'Heure du crime et le temps de l'œuvre d'art, Peter Sloterdijk nous donne à comprendre ce qu'est un moderne:

Est moderne celui qui est touché par la conscience du fait que lui ou elle, au-delà de l'inévitable qualité de témoin, est intégré par une sorte de complicité à ce monstrueux d'un type nouveau. Si l'on demande à un moderne: "Où étais-tu à l'heure du crime?», la réponse est: «J'étais sur le lieu du crime». Et cela signifie dans le périmètre de ce monstrueux global qui, en tant que complexe des circonstances modernes $d u$ crime. inclut ses complices par l'action et ses complices par le savoir. La modernité, c'est le renoncement à la possibilité d'avoir un alibi. (Sloterdijk, 2000: 10)

Ici, les survivants-victimes ne veulent en aucun cas avoir un alibi. Ils désirent être complices de l'exécution, complices du monstrueux et demandent à être sur les lieux du crime, comme s'ils ne l'étaient déjà pas assez. Il y aurait même ici une certaine fierté à y être et c'est peut-être ce que Sloterdijk nous amène à penser. Il faudrait penser ici le désir certain de participer au monstrueux. Si l'humain de nos jours est toujours présent sur le lieu du crime par l'intermédiaire des médias d'information, à toute heure du jour et de la nuit, les victimes d'Oklahoma City ont le droit d'y être encore davantage et de se donner pour acteurs de la scène. Du moins c'est ce que semble nous apprendre cette histoire.
Dix représentants des médias ont eu le droit d'assister dans une pièce adjacente à la diffusion de la mise à mort. Il y a eu une véritable mise en scène du dispositif de diffusion privée des images de la mort, mise en scène où rien du spectacle n'a été laissé au hasard. Des traiteurs ont très tôt été approchés afin de nourrir les 1400 reporters et photographes que l'on attendait sur les lieux de l'exécution et qui ont pu voir non pas l'exécution, mais la préparation de celle-ci et les témoins qui, eux, l'ont vue.

\section{IMAGES, DEUIL ET RÉGRESSION}

Cet appareil complexe mis en place par les instances juridico-politiques est pensé de telle sorte qu'un deuil soit possible et que l'on puisse donner une fin («bring closure» est le terme employé en anglais) à la tragédie passée. L'image de la mise à mort de Tim McVeigh une fois digérée par le spectateurvictime permettra à celui-ci de retrouver la paix et de ne plus être hanté par les images de l'explosion d'Oklahoma City. Là encore, on peut se demander pourquoi le monde entier n'a pas quant à lui le droit à être «libéré» de ce qui pourrait le hanter. Les images de l'immeuble éventré puis de l'immeuble dynamité par les autorités n'ont-elles pas fait le tour du monde? On peut parier que l'image qu'on a vue dans les médias est celle des victimes devenues elles-mêmes un peu bourreaux de leur bourreau. Le spectacle ne consiste pas en la mise à mort de McVeigh, mais consiste plutôt dans le dispositif de revanche où McVeigh n'est plus celui qui regarde la mort de ses victimes, mais où les places ont été interverties, tout simplement. À l'image d'un immeuble éventré de cris, de fumée et de pleurs, avec en arrière-plan l'esprit maléfique de celui qui a perpétré le crime, doit se substituer l'image de la mort de McVeigh vue par ses victimes. Dans cet espace, l'espace de l'image cadrée sur les victimes devenues bourreaux, le monde entier, bien sûr, ne fait, quant à lui, le deuil de rien, et surtout pas des morts. Mais les victimes elles-mêmes pourront-elles davantage faire le deuil de leurs morts ou de leurs blessures? Le procureur général a promis de faire en sorte que les victimes puissent clore ce 
chapitre de leur vie et veut apporter une fin à cette histoire. L'accomplissement du deuil est de toute évidence ce que la machine américaine de justice veut donner à voir et la production d'images de la mort de McVeigh n'a d'autre but que de donner à voir comment l'État américain permet aux spectateurs de ne pas rester dans la mélancolie. On pense que l'image de la mise à mort de McVeigh pourra parvenir à chasser les images qui hantent l'esprit des victimes. Le 19 avril dernier, tous les médias du pays étaient tournés vers le Mémorial d'Oklahoma City où sont inscrits les noms des victimes de l'attentat. La mort de McVeigh devrait permettre d'en finir avec cette histoire. Comme le dit le procureur général sur CNN:

Don't expect a wonderful feeling immediately overnight. Expect to be dealing with it for a while. But ultimately, if they feel going into this that it will help them resolve it, it probably will.

Ce qui est prévu, c'est un effet cathartique du spectacle, un effet de guérison, à long terme. Or, cette pensée d'une guérison par l'image de la mort du bourreau est peut-être le contraire de ce qu'il fallait pour que le deuil soit fait. Une image, celle de la mort de McVeigh, permettrait aux victimes de digérer l'affaire, de digérer les images qui leur sont restées à l'esprit pendant cinq ans. Une image permettrait de sortir de la haine, de quitter l'objet perdu qui hante les victimes et les proches et d'avaler une fois pour toutes les scènes qui ne cessent de se répéter. Mais cette idée d'une image réparatrice est bien sûr hautement problématique. Freud a mis l'arrachement au voir comme initiation de la pensée. Pontalis, dans un article intitulé "Perdre de vue», nous explique que cet

[...] arrachement [est] toujours à réeffectuer tant l'attraction par l'image ne cesse d'être active. Et nous, en plaçant le fauteuil derrière le divan, nous donnons une forme concrète à cette division du regard et de la pensée. Nous instituons la perte de vue comme condition de la pensée. (Pontalis, 1987 : 233-234)

Il me semble qu'il faut penser la perte de vue comme condition de la pensée et comme condition du deuil. En effet, ce qui est en jeu dans l'image, c'est son pouvoir de séduction, sa capacité à méduser le sujet. Comme Pontalis l'indique dans l'article que je viens de citer, il y a chez Freud une réelle fascination pour l'image qui mérite sans cesse d'être dépassée. Le rapport du visuel à l'inconscient est essentiel et non accidentel. Dans l'analyse que Pontalis fait de l'image chez Freud, la voie "régrédiente» qu'emprunte le rêve est à la fois régression vers l'image visuelle et attraction par le refoulé.

Il faut en quelque sorte mettre en scène cette fascination de l'image pour parvenir selon Freud à la dépasser et à créer une élaboration verbale. S'il y a chez Freud presque une régression visuelle dont le rêve serait le principal acteur, cette régression est néanmoins nécessaire pour parvenir à la mise en mots. Il ne peut y avoir évitement de l'image.

C'est précisément la question de la fascination qui est en cause dans l'exécution de McVeigh. En effet, aux images de l'attentat doit se superposer celle de la mort, de telle sorte que cette dernière image efface celles, horribles, d'un immeuble plastiqué et de corps disloqués, calcinés. À l'image de l'horreur doit succéder l'image d'une autre horreur.

Or, ce besoin de mettre en images comme moment régressif est peut-être nécessaire à la psyché. Il est peutêtre important que les victimes aient une image à partir de laquelle elles pourront se détacher et se mettre à penser et à faire le deuil. La mise en images est peut-être un moment nécessaire pour rendre compte de la folie de l'événement passé. Mais, dans le cas qui nous intéresse, cette mise en images ne permet pas au sujet de sortir de sa fascination médusée pour les images de l'horreur.

\section{MetTRe Fin À LA MÉLANCOLIE? \\ La victime comme sujet rejoue simplement sa propre terreur dans l'image de la mort de McVeigh. Ce sujet désire voir ce que c'est mourir, ce que lui a failli vivre et ce que les proches des morts imaginent chaque jour à propos de leurs amis ou de leurs parents disparus dans l'attentat. Par McVeigh et sa mort, les gens veulent re-voir la terreur du mourir et la dépasser. Mais cette terreur qui a été vue ou non n'a pas nécessairement mis fin à la mélancolie. De cette mise}


en images, il n'est pas du tout sûr que le deuil est venu ou viendra, parce que ce qui restera inanalysé dans cette folie scopique, c'est la fascination première pour les images de l'immeuble éventré, les corps disloqués ou encore pour McVeigh agonisant. Ce qui ne peut se penser dans la répétition des images de l'horreur, c'est précisément la fascination pour les images de la mort de $\mathrm{McVeigh}$, qui n'a rien à voir avec une quelconque réparation ou encore avec l'introjection nécessaire à tout deuil. Les victimes dans le spectacle de la mort de McVeigh vont simplement remplacer la vision fascinée qu'ils ont eue de la folie du mourir par celle d'une mort qu'ils croient contrôler. En fait, aux images folles de mort chaotique dans l'immeuble, on veut faire succéder l'image d'une mort rationnelle, préparée, propre. Les victimes n'auront pas accès à cette folie du mourir, parce que précisément ceux qui assisteront à cette scène veulent contempler en elle le caractère logiquement raisonnable qu'on veut lui donner. Il faut dire que toutes les victimes - ou proches des victimes vont devoir assister à une séance d'information où il va leur être expliqué ce qu'elles vont voir, ou espérer voir durant l'exécution. Les images qu'elles vont visionner vont leur être racontées à l'avance. Le côté mise en scène de cette exécution est mis en évidence dans le discours du gardien en chef de la prison de Haute Terre, Indiana, où aura lieu l'exécution. Celui-ci donc ressasse sans cesse aux médias que lui et son équipe font des répétitions de l'exécution: «We want to make sure this goes very, very well». Ils se conformeront pour y arriver à ce fameux protocole fédéral de 56 pages dont j'ai parlé plus haut afin que la chose soit conduite "in a efficient and human manner». On prévoit des applaudissements à la fin, comme cela est souvent le cas dans les exécutions orchestrées par ceux de ces états américains où la peine de mort est appliquée de façon fréquente. Rien dans cette mise à mort et dans sa diffusion ne sera laissé au hasard. Il ne s'agit donc pas de mettre en scène le chaos de la mort tel qu'il s'est présenté aux yeux des victimes et du monde entier lors de l'attentat ou tel qu'il pourrait se présenter dans les yeux d'un condamné à mort, mais bien de faire de l'image de la mort une image rationnelle, préconstruite.
Le gouvernement américain est donc en train de préparer un deuil préfabriqué. Il fait dans le prêt-àporter du deuil et permettra aux victimes d'effacer les images de la mort comme folie au profit de celles de la mort comme raison. Il n'y aura pas de réelle traversée de la fascination pour l'horreur. En ce sens, le policier qui a ouvert un site électronique, dans lequel il reproduit 88 images de l'attentat d'Oklahoma $\mathrm{City}^{2}$, se situe peut-être davantage à l'intérieur d'un deuil que ceux qui veulent assister à l'exécution. En effet, à travers ces images où lui-même se donne à voir dans la photo numéro un, le policier met en scène sa fascination pour l'iconographie de l'attentat. Il met en scène son regard halluciné par la mort, et en ce sens passe et repasse à travers cette position médusée qui est la sienne. Mais dans l'ordre maniaque donné aux 88 photos, il y a aussi une tentative désespérée de mettre de l'ordre dans les images de la mort. Contre quoi le visuel de l'exécution se construit-il? En fait, c'est contre la tentation de sombrer dans une folie des images, dans leur surabondance, leur horreur, dans leur perte de sens et dans leur effet de déréalité. Ce que les victimes d'Oklahoma City vont faire, ce n'est pas le deuil de la scène et des morts: elles vont assister à la production imagière d'une logique de la mort, d'une logique de mort. Le monde entier, lui, qui ne verra pas la mort de McVeigh, se contentera de voir la production du film de sa mort. Le monde entier devra en quelque sorte se contenter du «making of» du deuil collectif.

\section{N O TES}

1. On n'a qu'à penser au traitement médiatique que le père de $\mathrm{McVeigh} \mathrm{reçoit} \mathrm{où} \mathrm{il}$ est présenté comme la victime de son fils, la victime de sa paternité. Le père McVeigh déclare d'ailleurs sur les chaînes de télévision qu'il a hâte que son fils soit exécuté et que cela se termine, cette histoire...

2. (http://www.efaubian.com/ bomb/ bomb.html).

\section{RÉFÉREN CES BIBLIO G RAPHIQ U ES}

AdoRno, T.W. [1980] : Minima Moralia, Paris, Payot.

Hegel, G. F.W. [1979]: Introduction à l'esthétique. Le Beau, Paris, Flammarion.

KOJĖVE, A. [1947]: Introduction à la lecture de Hegel, Paris, Gallimard.

LYOTARD, J.-F. [1983] : Le Différend, Paris, Minuit.

PONTALIS, J.-B. [1987] : «Perdre de vue», dans Nouvelle Rerue de psychanalyse, nº35, printemps, Paris, Gallimard.

SLOTERDiJK, P. [2000]: L'Heure du crime et le temps de l'œuvre d'art, Paris, Calmann-Lévy. Tisseron, S. [1997]: Psychanalyse de l'image. Des premiers traits au virtuel, Paris, Dunod. ViRno, P. [1999]: Le Souvenir du présent. Essai sur le temps historique, Paris, Éd. de l'Éclat. 